\title{
Filamentary Structures Near the Galactic Center
}

\author{
F. Yusef-Zadeh ${ }^{1}$ \\ LASP, Goddard Space Flight Center and Northwestern University
}

\begin{abstract}
Recent studies of the Galactic center environment have revealed a wealth of new thermal and nonthermal features with unusual characteristics. A system of nonthermal filamentary structures tracing magnetic field lines are found to extend over $200 \mathrm{pc}$ in the direction perpendicular to the Galactic plane. Ionized structures, like nonthermal features, appear filamentary and show forbidden velocity fields in the sense of Galactic rotation and large line widths. Faraday rotation characteristics and the flat spectral index distributions of the nonthermal filaments suggest a mixture of thermal and nonthermal gas. Furthermore, the relative spatial distributions of the magnetic structures with respect to those of the ionized and molecular gas suggest a physical interaction between these two systems. In spite of numerous questions concerning the origin of the large-scale organized magnetic structures, the mechanism by which particles are accelerated to relativistic energies, and the source or sources of heating the dust and gas, recent studies have been able to distinguish the inner 200pc of the nucleus from the disk of the Galaxy in at least two more respects: (1) the recognition that the magnetic field has a large-scale structure and is strong, uniform and dynamically important; and (2) the physics of interstellar matter may be dominated by the poloidal component of the magnetic field.
\end{abstract}

\section{Background}

The proximity of the nucleus of our galaxy gives us an unparalleled opportunity for studying the detailed structure of the core of a spiral galaxy. Since the extinction of light by dust and gas is greater than 28 magnitudes, much of our understanding of this region comes from information revealed by radio, infrared, $\mathrm{X}$-ray and $\gamma$-ray observations. As a result of this rich body of information, the Galactic center region is differentiated into many complex components whose physical relationships are yet to be determined. Two such complex structures are the radio continuum Arc which lies at $1 \sim 0.2^{\circ}$ and the "omega-shaped" structure which extends over $1^{\circ}$ away from the Galactic plane and appears to engulf the Galactic center. A close examination of these two sources are essential for a better understanding of the environment of the Galactic center region on a scale of 200pc.

The subject of much of this review is the recent results on the continuum Arc and the "omegashaped" structures. After a short historical background, the filamentary structures found in the Arc, Sgr C and G359.54+0.18 are described including their polarization and their spectral index distributions. In section III, the characteristics of a number of thermal sources within the Arc, G0.2+0.05, G0.1+0.08, G0.18-0.04, and G0.15-0.05 are described, and arguments for their possible interaction with the nonthermal filaments are presented. Finally, the implications of recent observations are discussed, including a brief overview of theoretical attempts to explain the origin of radio structures near the Galactic center.

1 National Research Council Resident Research Associate 
The radio continuum Arc was first discovered during the continuum observations made by Drake (1959, see Steinberg and Lequeux) using the 85-foot telescope of the NRAO. His first two-dimensional map of the Galactic center region resolved the inner degree of the Galactic center into four components. This map, which has a resolution of $6^{\prime}$ at $\lambda 3.7 \mathrm{~cm}$ is reproduced in Figure 1 , shows the $\mathrm{Sgr} \mathrm{A}$ complex joined by a hook-shaped radio continuum Arc. The two extended components to the north and south, aligned in the direction of the Galactic plane, are usually referred to as the Sgr B and Sgr C HII complexes, respectively.

The complexity of the large-scale radio continuum emission of the inner $5^{\circ}$ of the Galaxy (Figure 2) can be seen in the survey made at $\lambda 11 \mathrm{~cm}$ (Altenhoff et al. 1978). A large-number of discrete sources concentrated mainly along the Galactic plane, plus the extended structures away from the plane demonstrate the wide range of angular scales associated with radio structures in this region.

The most notable extended features are the broad extensions of the continuum Arc at $l \sim 0.2^{\circ}$ and $\mathrm{Sgr} \mathrm{C}$ at $\mathrm{l} \sim-0.6^{\circ}$ to latitudes as high as $1^{\circ}$. High-frequency observations made by Kapitzky and Dent (1974) showed a lack of large-scale extended emission at $\lambda 2 \mathrm{~cm}$ and, therefore, suggested the nonthermal characteristics of these broad features.

The first comprehensive radio recombination line study of the continuum Arc, using single-dish telescopes, showed clearly that a large portion of this object, specifically the northern half of the Arc at positive latitudes, has thermal characteristics (Pauls et al. 1976; Whiteoak and Gardner 1973; Pauls and Mezger 1980). The recombination line intensity maps by Pauls and Mezger illustrated two regions of significant line emission within the Arc (G0.18-0.04 and G0.1+0.08) and no line emission was detected from the southern half of the Arc at G0.16-0.15 indicating nonthermal emission. A similar conclusion was also reached from the comparison of far-IR and radio continuum emission from the Arc (Dent et al. 1984; Brown and Liszt 1984). Additionally, Güsten and Downes (1980) determined the electron temperature distribution from recombination line observations and pointed out that a sizable fraction of the continuum emission from G0.18-0.04 could be nonthermal. Based on $\mathrm{H}_{2} \mathrm{CO}$ and $\mathrm{HI}$ absorption studies, only the front side of the "molecular ring" is seen in absorption against the lower half of the Arc near G0.16-0.15 (Güsten and Downes 1980; Lasenby et al. 1988). This suggests the continuum Arc is located within the inner 200pc of the Galactic center region.

\section{Nonthermal Filaments}

\section{a)Morphological Details}

High-resolution radio continuum observations of the continuum Arc using the VLA have produced a collection of totally unexpected filamentary structures (Yusef-Zadeh, Morris and Chance 1984; Yusef-Zadeh and Morris 1987a [hereafter YM87a]). The linear segment of the Arc, as shown in the $\lambda 20$ and $\lambda 6 \mathrm{~cm}$ radiographs of Figures $3 \mathrm{a}, \mathrm{b}$, was recognized to consist of a group of straight and narrow filaments which are coherent over $40 \mathrm{pc}$, have a typical width of $\sim 1 \mathrm{pc}$ and run parallel to each other in the direction perpendicular to the Galactic plane. The adjacent filaments appeared to have helicity and twist about each other. A new picture of the Galactic center Arc indicates that the three prominent components G0.18-0.04, G0.16-0.15 and G0.1+0.08 are all an integral part of an organized structure, rather than chance superposition of a number of compact sources as had been suggested previously. The observations made with the 100-m Bonn telescope (Seiradakis et al. 1985) indicate that the linear segment of the Arc extends far beyond the 40pc filametary structure found in the VLA observations (see also Tsuboi et al. 1986).

Recent observations by Morris and Yusef-Zadeh (1985) have drawn attention to another type of filamentary structure, "threads", found near the Galactic center. The morphology of these remarkable thread-like structures are distinguished from the group of filaments in the Arc and the 


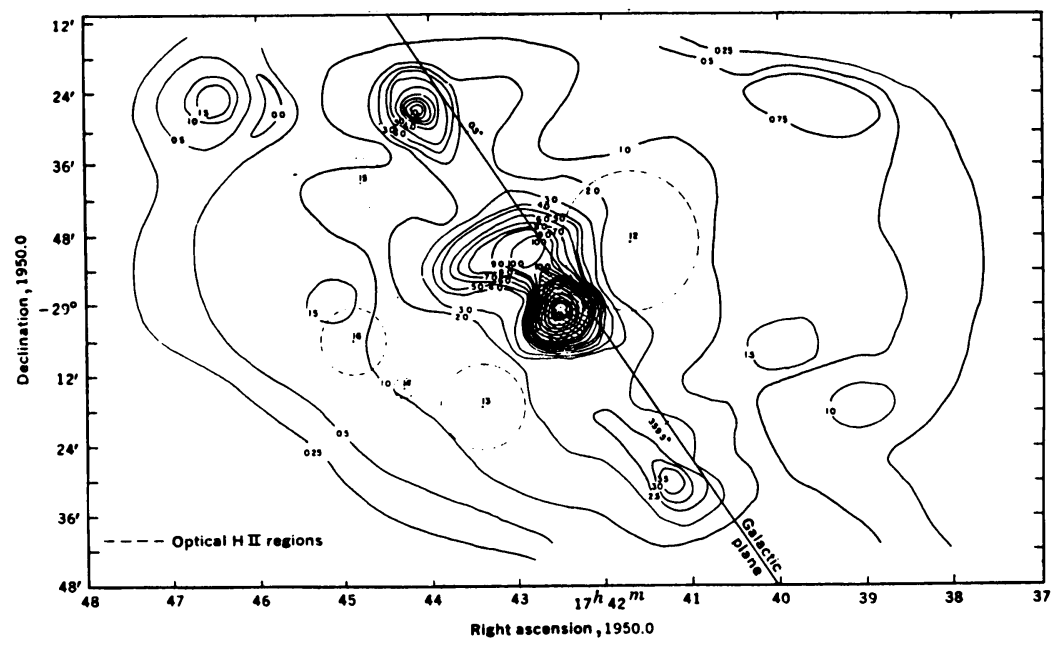

Figure 1: Contour map of the inner 200pc of the Galaxy made by Drake (1959) at $8 \mathrm{GHz}$ with the spatial resolution of $6^{\prime}$.

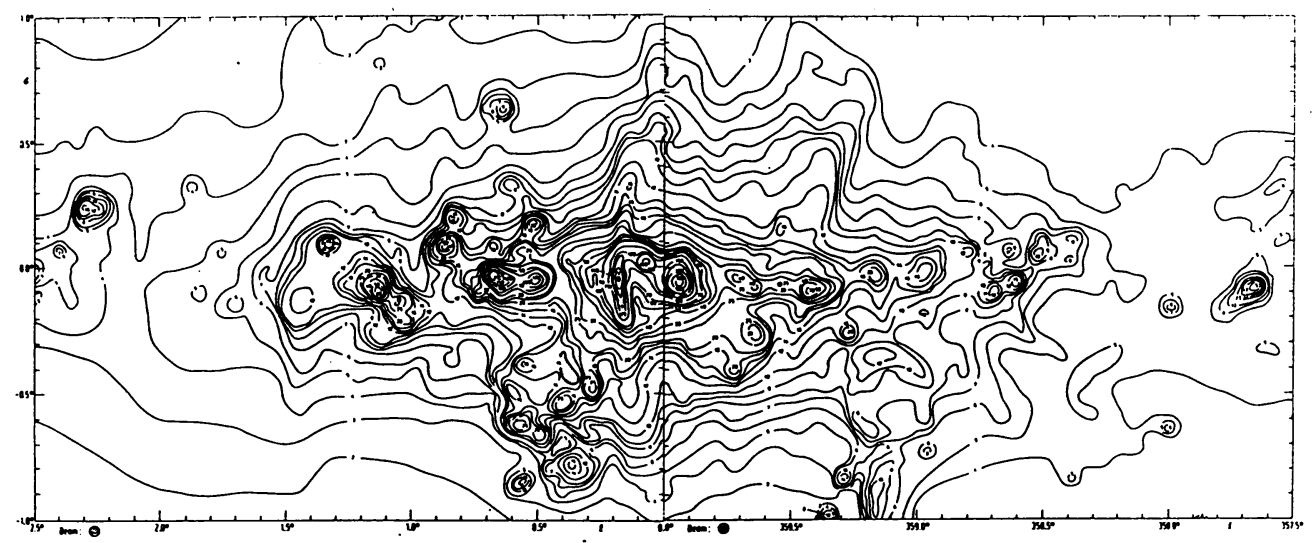

Figure 2: Contour map of the inner $5^{\circ} \times 2^{\circ}$ of the Galaxy at $4.8 \mathrm{GHz}$ made with the $2.6^{\prime}$ beam (Altenhoff et al. 1978).

filamentary structure found in Sgr C (Liszt 1985) only in that they are isolated from ionized gas, appear one-dimensional and have a uniform surface brightness along their lengths. Other examples of "threads" have been detected, all of which are located either in the "omega-shaped" structure (see below) or in the vicinity of the Arc and its extensions. In Figures 4(a-c), we display the morphology of several "threads" all embedded in large-scale diffuse gas near the Galactic center:

Figure 4a shows two of the most prominent "threads" which extend over $10^{\prime}$ in the direction almost perpendicular to the Galactic plane. The long northern and southern "threads" cross the thermal structures associated with the Arc and the Sgr A complex, respectively. A small thread-like structure 


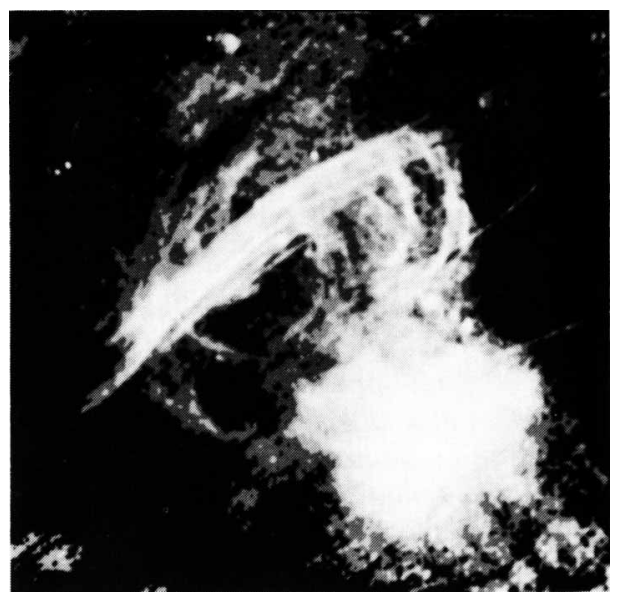

Figure $3 a$ : The $\lambda 20 \mathrm{~cm}$ radiograph of the inner $30^{\prime}$ of the Galactic center showing the radio continuum Arc and the Sgr A complex with a spatial resolution of $5^{\prime \prime} \times 8^{\prime \prime}(\alpha \times \delta)$.

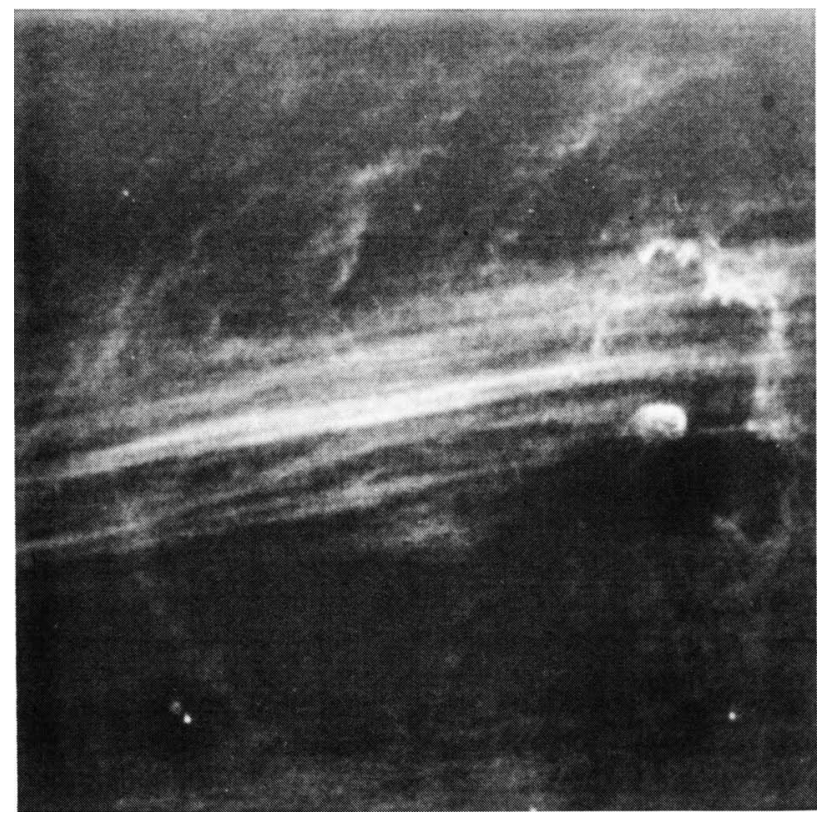

Figure 3b: The $\lambda 6 \mathrm{~cm}$ radiograph of the region near G0.16-0.15, shown in Galactic coordinate system, with a spatial resolution of $3^{\prime \prime}$. The long straight filaments run in the direction almost perpendicular to the Galactic plane. 
is also noted at the western edge of Figure 4a. all these features are linearly polarized (Morris and Yusef-Zadeh 1989)

Figure $4 \mathrm{~b}$ shows a few cases of thread-like structures found in the $\lambda 20 \mathrm{~cm}$ radiograph of the southern extension of the Arc (Yusef-Zadeh et al. 1989). A similar distribution of weakly emitting thread-like structures has also been noted by Liszt (private communication) in the region surrounding $\mathrm{Sgr} \mathrm{C}$. The bright extended component at the western edge of this image coincides with G0.16-0.15 in the Arc (see Fig. 6b). The straight filaments at higher negative latitudes show an inclination between $\sim 20^{\circ}$ and $50^{\circ}$ with respect to the Galactic rotation axis.

Figure 4c depicts another pair of isolated "threads" lying at G359.54+0.18 (Bally and Yusef-Zadeh 1989). They are situated $15^{\prime}$ to the north of $\mathrm{Sgr} C$, run parallel to each other with an inclination of $22^{\circ}$ and appear to have the same orientation as that of the negative longitude side of the "omega-shaped" feature. No obvious connection is seen between the straight filaments in Sgr C and G359.54+0.18.

Sofue and Handa (1984) recognized a large-scale "omega-shaped" structure which extends in the direction away from the Galactic plane and toward positive latitudes. Figure 5 shows the composite images of the Arc, the "omega-shaped" structure, Sgr C and G359.54+0.18. The positive-longitude side of the "omega-shaped" structure represents the low-intensity northern extension of the group of filaments which form the Arc. This picture shows clearly that the scale of the phenomenon producing the Arc structure is $\sim 200$ pc directed perpendicular to the plane of the Galaxy. Sgr C is another complex source which consists of a set of filamentary structures oriented in the direction almost perpendicular to the Galactic plane (Liszt 1985). The linear filaments in Sgr C are located at the base of the negative-longitude side of the "omega-shaped" structure and are surrounded mainly by a ring-like ionized gas with a central velocity of $\sim-60 \mathrm{~km} \mathrm{~s}^{-1}$ (Odenwald 1988; Anantharamaiah and Yusef-Zadeh 1988). G359.5+0.18 lies at the eastern edge of the negative-longitude side of the "omega-shaped" structure (Bally and Yusef-Zadeh 1989).

\section{b) Polarization Characteristics}

Many of the straight filaments found near the Galactic center, whether they are grouped together like the Arc and Sgr C or are relatively isolated from ionized gas like "threads" and G359.54+0.18, emit linear polarization at radio wavelengths, and therefore, their radiation mechanism is probably synchrotron emission (Yusef-Zadeh and Bally 1989; Morris and Yusef-Zadeh 1989). The nonthermal nature of the straight filaments in the Arc is best shown in Figure 6a where polarized emission arises from a pair of adjacent filaments near G0.16-0.15 at $\lambda 2 \mathrm{~cm}$ (Yusef-Zadeh 1986; Inoue et al., 1988). In Figure $6 \mathrm{~b}$, the total intensity radiograph at $\lambda 6 \mathrm{~cm}$ shows a larger area than that shown in Figure $6 \mathrm{a}$ and exhibits a complex region composed of a bundle of polarized filaments running parallel to each other. This morphology is remarkably similar to that of aurora streamers.

High-resolution polarization measurements show a significantly different distribution of polarized emission at different wavelength bands, and therefore, the intrinsic direction of the magnetic field lines along individual narrow filaments has not been determined accurately. Two effects are responsible for a decrease in the degree of polarization: One is Faraday rotation of the polarization angle which is directly proportional to $\lambda^{2}$ and occurs when the electromagnetic wave propagates through a magnetized medium lying either internal or external to the source of nonthermal radiation. The other important effect is the rotation of the wave across the resolution element.

Single-dish polarization measurements have accounted for the effect of Faraday rotation and have determined that the large-scale distribution of the magnetic field geometry is directed along the rotation axis of the Galaxy (Tsuboi et al. 1986; Reich 1988). Figure 7, which is based on observations made with the Nobeyama radio telescope, shows the two polarized plumes to coincide 
Figure 4a: The $\lambda 20 \mathrm{~cm}$ radiograph of the western segment of the continuum Arc showing the long thread-like structures.

Figure $4 b$ : The $\lambda 20 \mathrm{~cm}$ radiograph of the eastern segment of the continuum Arc showing a number of thread-like structures along the southern extension of the Arc.
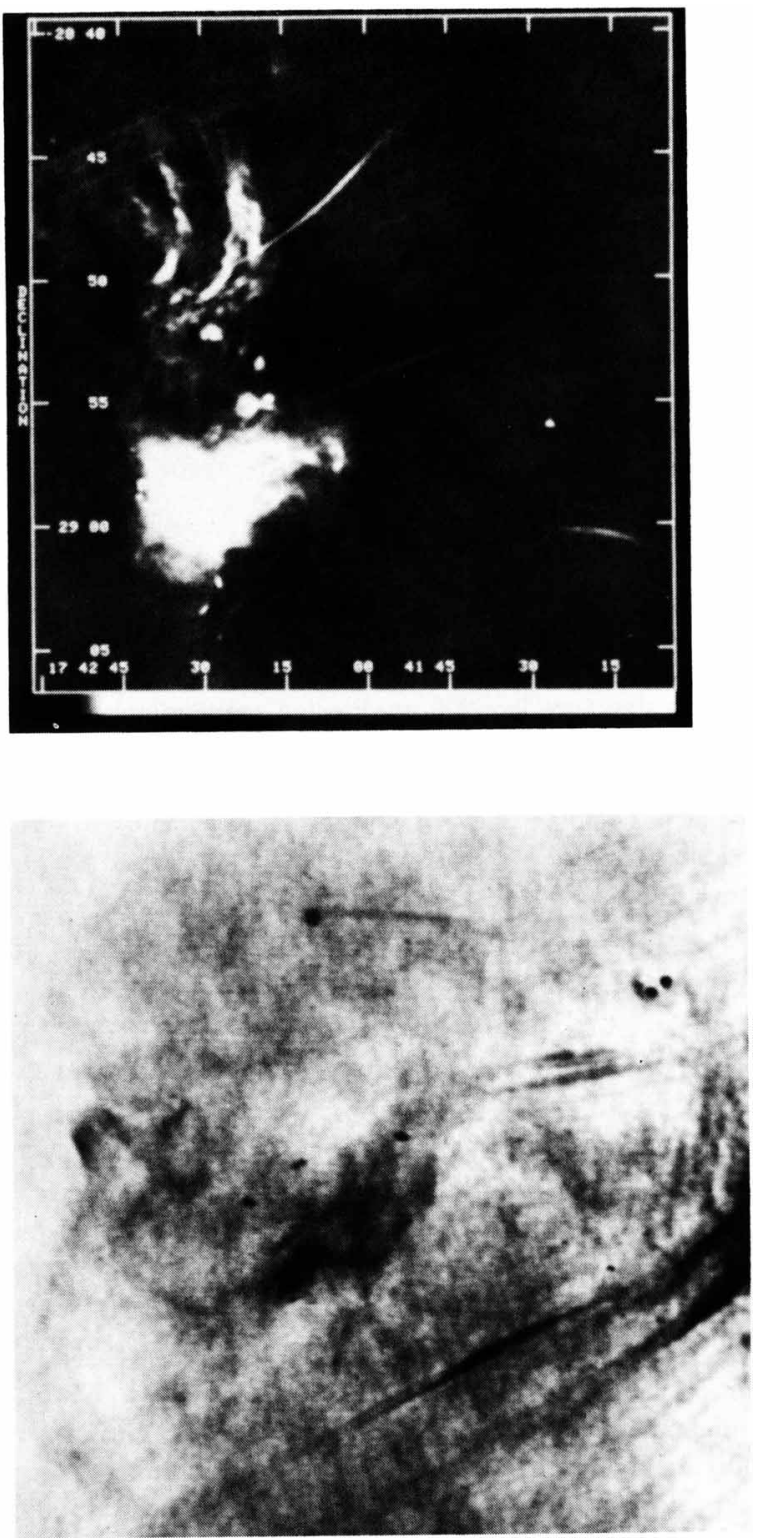
Figure 4c: The total intensity radiograph of G359.54+0.18 with a resolution of $10^{\prime \prime}$ at $\lambda 6 \mathrm{~cm}$.
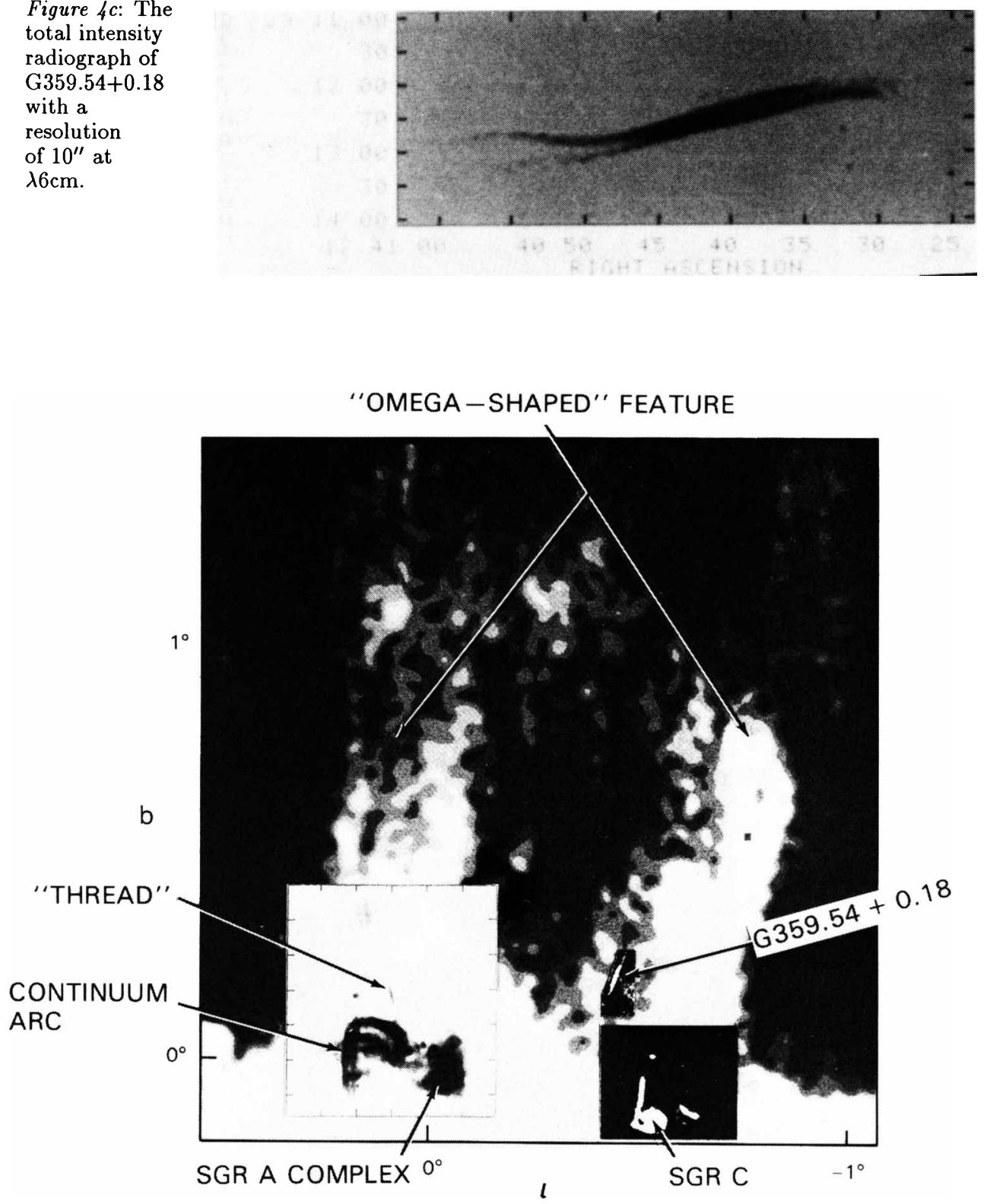

Figure 5: The radio images of the continuum Arc (YM88), Sgr C (Liszt 1985) and G359.54+0.18 (Bally and Yusef-Zadeh 1989), all based on VLA measurements, are superimposed on the large-scale radiograph of the "omega-shaped" structure taken from Sofue and Handa (1984). 

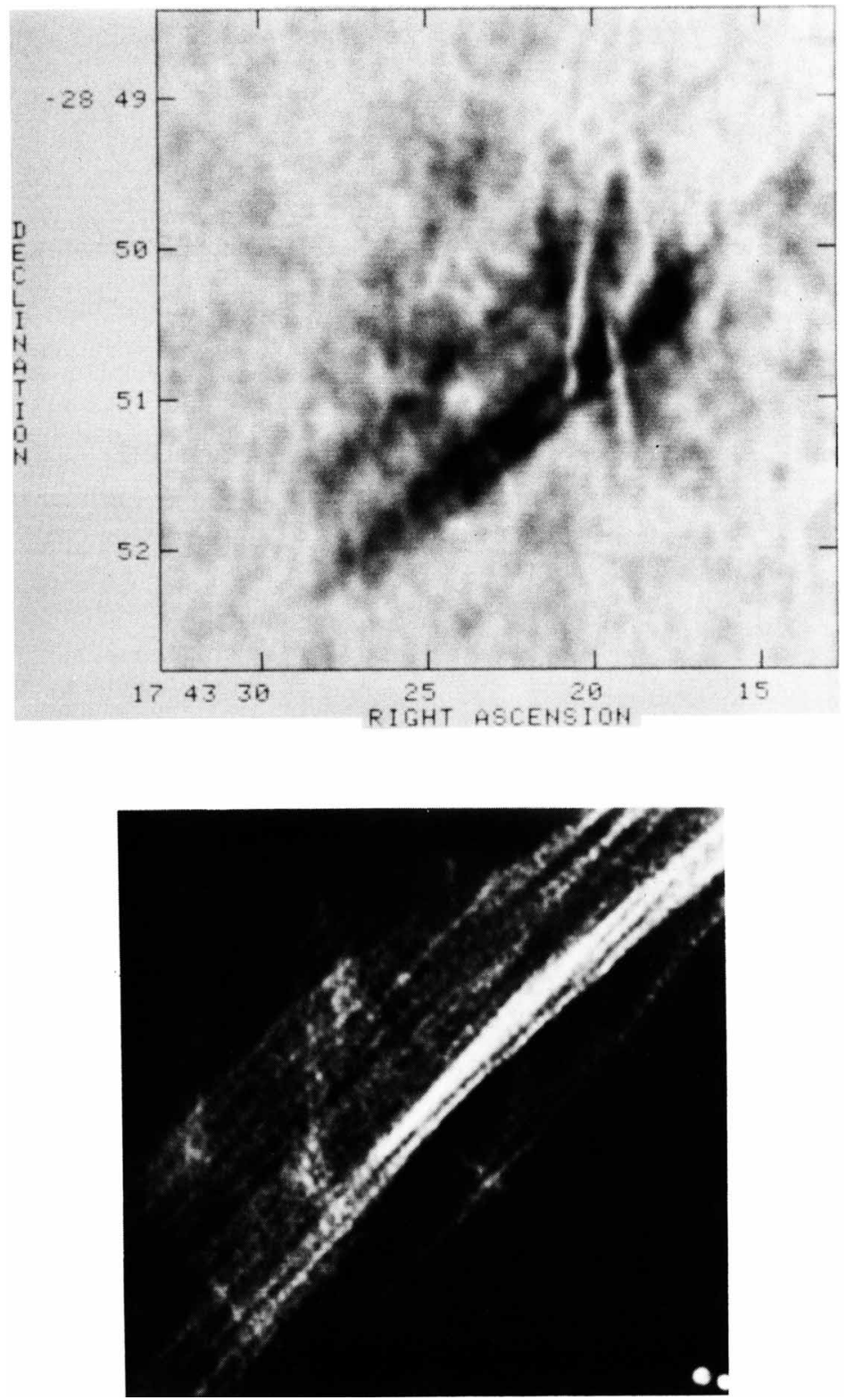

Figure 6a,b: (top) The $\lambda 2 \mathrm{~cm}$ polarization image of the region near G0.16-0.15 with a resolution of $5^{\prime \prime}$. (bottom) The $\lambda 6 \mathrm{~cm}$ total intensity radiograph of G0.16-0.15 showing an area $5^{\prime} \times 5^{\prime}$. 
with the northern and southern extensions of the Arc (see the total intensity counterpart of the polarized plumes in Figure 3). The line segments indicate the direction of the magnetic field lines which appear to be predominently oriented perpendicular to the Galactic plane (Tsuboi et al. 1986). The distribution of the rotation measure in the polarized plumes, as shown in Figure 7, indicates a reversal of the line-of-sight component of the magnetic field in the opposite side of the Galactic plane. This inverted distribution of the rotation measure is attributed to the bending of the largescale magnetic field lines by the disk rotation as the field lines cross the Galactic plane (Sofue et al. 1987). The percent polarization of the northern and southern plumes which appear to be centered symmetrically with respect to the compact source (G0.16-0.15) is $\sim 40 \%$ and $\sim 15 \%$, respectively, and therefore, the field lines are inferred to be highly organized. The radio luminosity of the plumes and the magnetic field strength based on the equipartition assumption are estimated to be $\sim 10^{35}$ $\mathrm{erg} \mathrm{s}^{-1}$ and several tens of $\mu \mathrm{G}$, respectively (Tsuboi et al. 1986).

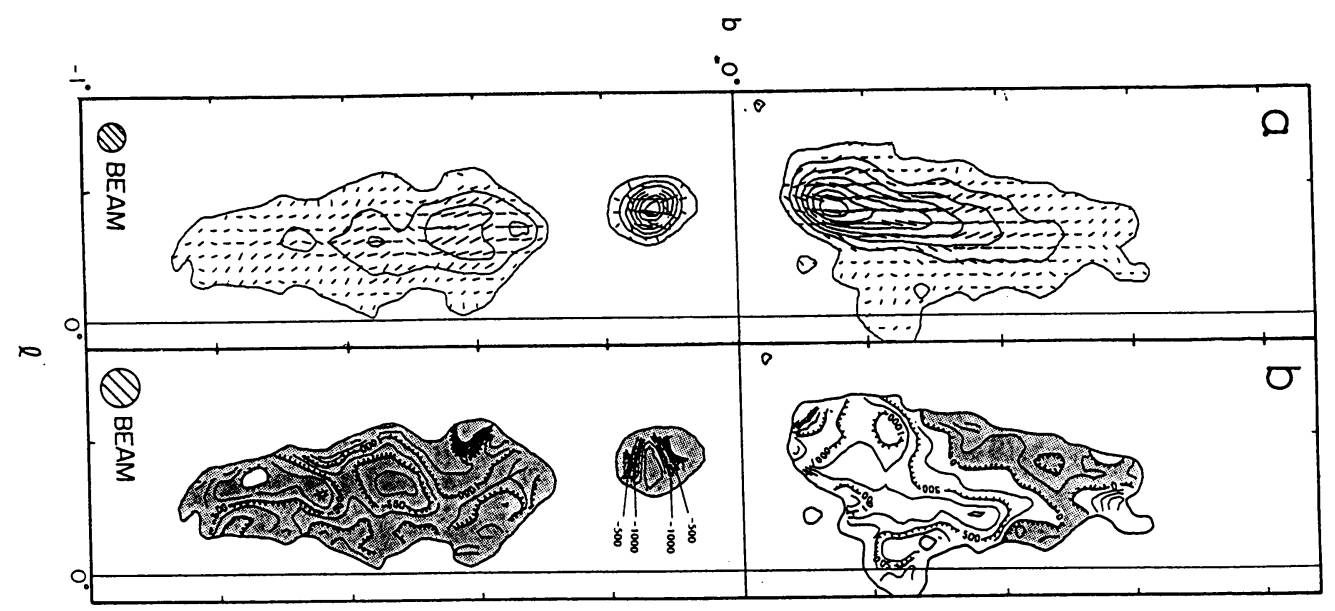

Figure 7: (top) The distribution of the magnetic field orientation is superimposed on contours of the polarized intensity from the continuum Arc and its two plume-like extensions away from the Galactic plane (Tsuboi at al. 1986). (bottom) The corresponding rotation measure (RM) distribution, with shaded areas presenting positive values of RM.

The negative-longitude side of the "omega-shaped" structure showed an absence of polarized emission at $\lambda 3 \mathrm{~cm}$ with a resolution of $3.5^{\prime}$ (Tsuboi et al. 1986). However, recent VLA polarization measurements of G359.54+0.18 (see Fig. 4c) showed a high degree of polarization at $8 \mathrm{GHz}$, ranging from $50 \%$ to $70 \%$ (Yusef-Zadeh and Bally 1989). This suggests that the magnetic field has a finescale structure and is highly organized in this region (Yusef-Zadeh and Bally 1989). The lack of polarized emission based on single-dish measurements is due to beam depolarization.

The lack of polarized emission at $\lambda 3 \mathrm{~cm}$ from the region between the two plumes and the compact source is attributed to the large Faraday rotation caused by the thermal medium surrounding the Arc. The $\lambda 2 \mathrm{~cm}$ polarization measurements which are less affected by the Faraday depolarization than those at $\lambda 3 \mathrm{~cm}$, show linear polarization from the region between the plumes (Inoue et al. 1988). This is further evidence in support of the association of the linear segment of the Arc and the two polarized plumes. 
A polarization anomaly in the region near G0.16-0.15 has been reported by Sofue et al. (1987) who compared the $5 \mathrm{GHz}$ and $10 \mathrm{GHz}$ polarization characteristics. This region is known to have a very high rotation measure ranging between +350 and $-5500 \mathrm{rad} \mathrm{m}^{-2}$, a high multiplicity of filamentary structure (see Fig. 6b), and the appearance of a helical feature winding around the filaments (Inoue et al. 1984; YM87a). Sofue et al. find a decrease in the degree of polarization with increasing frequency from this region. This is rather unusual behavior since the rotation measure decreases with increasing frequency and the effect of depolarization is reduced at high frequencies. Three possible suggestions have been proposed to explain this anomaly: a) The thermal and nonthermal gas are intermixed and the radiation is subject to Faraday rotation which varies with depth from where it emerges. In this idealized situation, a minimum in the degree of polarization occurs when the $\Phi \lambda^{2}$ ( $\Phi$ is the rotation measure) is a multiple of $\pi$ (Sofue et al. 1987; Burn 1966). Future polarization measurements at a number of nearby frequencies, preferably at $8 \mathrm{GHz}$, are needed to test this hypothesis; $b$ ) If the magnetic field lines along the straight filaments have a helical component, the superposition of different electric field directions emerging from different segments of the helically wound filaments could produce the observed polarization anomaly (Lasenby et al. 1988). c) If the spectrum of the continuum emission varies across the $2.5^{\prime}$ beam of the telescope, Lasenby et al. 1988 show that a change of 1.7 or greater in the spectral index distribution is required to account for the observed polarization behavior (Burn 1966). The flat spectral index distribution of the filaments, which is discussed next, does not support this last hypothesis.

\section{c) Spectral Index Distribution}

The radio continuum Arc has been observed at numerous frequencies ranging from $80 \mathrm{MHz}$ to 45GHz (Mills and Drinkwater 1984; Little 1974; Yusef-Zadeh et al. 1986; Kassim et al. 1987; Sofue et al. 1986; and the references cited therein). The hook-shaped appearance of the Arc is identified at all observed frequencies except at frequencies $\leq 160 \mathrm{MHz}$. At $160 \mathrm{MHz}$ and $80 \mathrm{MHz}$, only the southeastern portion of the Arc is visible (Yusef-Zadeh et al. 1986; Kassim et al. 1987). This region coincides with G0.16-0.15 where the polarized emission from the Arc has the largest rotation measure, perhaps the largest in the Galaxy. A nonuniform distribution of thermal plasma is thought to be responsible for absorbing the low-frequency emission and depolarizing the synchrotron radiation from the northern portion of the Arc. The electron density of thermal gas is estimated to vary by two orders of magnitude from $4 \mathrm{~cm}^{-3}$ to $500 \mathrm{~cm}^{-3}$ (Yusef-Zadeh et al. 1986).

At high frequencies, the spectral index distribution of the Arc and its extensions based on single dish measurements, shows a flat spectrum (Sofue and Handa 1984; Sofue et al. 1986) and is consistent with earlier measurements by Mills and Drinkwater (1984). At a spatial resolution of $10^{\prime \prime}$, the spectral index distribution, as displayed in Figure 8a, shows that the nonthermal filaments have a flat spectral index, $\alpha\left(\mathrm{F}_{\nu} \propto \nu^{\alpha}\right)$ ranging between -0.2 to 0.1 and therefore, in agreement with previous low-resolution measurements. The spectral index map, which is made by the comparison between the $\lambda 20$ and $6 \mathrm{~cm}$ (Fig. 8b) VLA maps, shows also the steep spectrum of Sgr A East and the nearly flat spectrum of Sgr A West which are consistent with previous measurements by Ekers et al. (1985). The $\lambda 6 \mathrm{~cm}$ map, as seen in Figure $8 \mathrm{~b}$ was obtained by combining 6 overlapping fields at $\lambda 6 \mathrm{~cm}$.

It should be pointed out that the single-dish measurements of the spectral index distribution across the Arc and its extensions are marred by a number of difficulties, such as the effect of background emssion from the Galactic disk at different frequencies and a large number of fine-scale thermal and nonthermal features that are not spatially resolved. The interferometric measurements generally lack the short spacing flux values and therefore cause uncertainties in the true distribution of large scale structures. In spite of these difficulties, there is a general agreement in all measurements that 

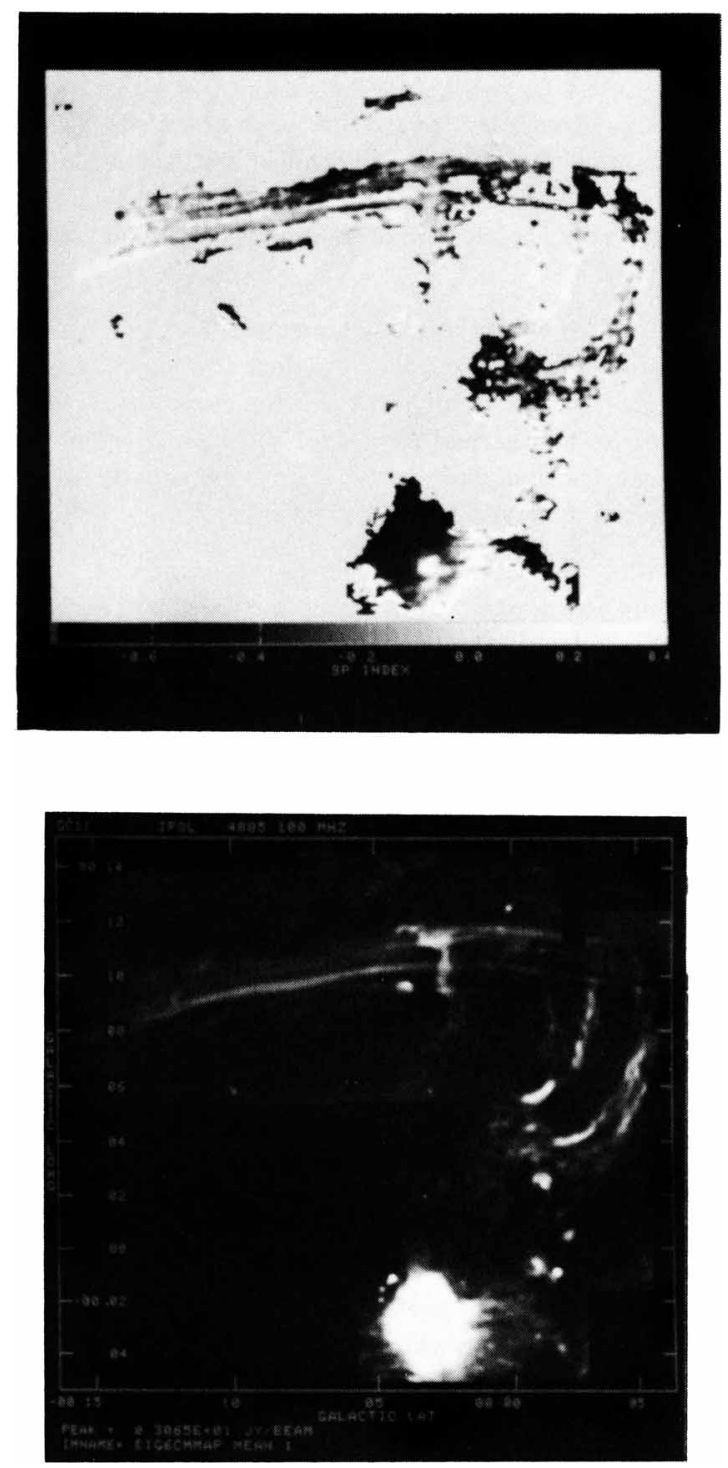

Figure 8a,b: (top) The spectral index distribution with a resolution of $10^{\prime \prime} \times 10^{\prime \prime}$ is derived by comparing the $\lambda 6$ and $20 \mathrm{~cm}$ images. The key at the bottom of this figure shows the range of spectral index values. The $\lambda 6 \mathrm{~cm}$ and $\lambda 20 \mathrm{~cm}$ data used to contruct this image were obtained by using the same (uv) coverage corresponding to observations made using the C/D and B/C array of the VLA, respectively. (bottoom) The $\lambda 6 \mathrm{~cm}$ image constructed by combining six overlapping fields at $\lambda 6 \mathrm{~cm}$. The top and bottom figures cover exactly the same area. 
the nonthermal features near the Arc have a flat spectral index. Three possible explanations are proposed for this unusual behaviour of the spectral index distribution.

a) If the thermal gas is mixed with the relativistic particles along the filaments, models of the resultant spectrum are found to be flat (Sieber and Schlickeiser 1982). This picture is not inconsistent with the polarization characteristics of the Arc and the relatively steep spectrum of G359.54+0.18 (Yusef-Zadeh and Bally 1989), another system of nonthermal filaments, which is not associated with any ionized medium.

b) If the flat spectral index of the nonthermal filaments is a consequence of a low-energy cut-off in the power law distribution of energetic particles, then the loss of the low-energy particles (see next section) might be explained as a result of their large cross section in ionizing the thermal gas which appears to surround the nonthermal structures. A recent recombination line survey with a spatial resolution of $2^{\prime}$ illustrates that much of the diffuse gas associated with the Arc has thermal characteristics (Anantharamiah and Yusef-Zadeh 1988).

c) The third possiblity is that the electron distribution has a monoenergetic distribution and that the flat spectrum reflects the young age of the relativistic particles (Sofue et al. 1986).

\section{Thermal Structures}

It is generally possible to distinguish thermal and nonthermal features by their spectral characteristics. However, the flat spectral index of the linearly polarized emission from the Arc and the "omega-shaped" structure, has made this technique unreliable. Recently, Reich et al. (1988) devised a method to separate thermal and nonthermal structures near the Galactic center by comparing the ratio of $60 \mu \mathrm{m}$ flux density to the $\lambda 11 \mathrm{~cm}$ flux density. Although this technique is applicable for large-scale extended structures, a number of complementary measurements such as molecular and recombination line observations are needed to unravel the fine-scale thermal components. Some of these measurements are described below.

a) $G 0.2+0.05$

The H78 $\alpha$ and $\mathrm{H} 98 \alpha$ recombination line survey has recently been carried out over the inner $40^{\prime}$ of the Galactic center region using the $43 \mathrm{~m}$ telescope at Green Bank (Anantharamaiah and YusefZadeh 1988). This survey detected thermal emission from most of the Arc, the Sgr A complex and the region between these two prominent features. The complex structure centered at G0.2-0.05 is seen to have thermal characteristics having velocities ranging between $15 \mathrm{~km} \mathrm{~s}^{-1}$ to $35 \mathrm{~km} \mathrm{~s}^{-1}$. The high-resolution continuum structure of G0.2-0.05 is identified in Figure 9 as a semi-circular loop-like structure lying to the north of the straight portion of the Arc. The kinematics of the ionized gas near the Arc gives some support to the HI data which suggest that the $20 \mathrm{~km} \mathrm{~s}^{-1}$ molecular cloud may be associated with the Arc (Lasenby et al. 1988). A stronger correlation between the ionized gas and the $-50 \mathrm{~km} \mathrm{~s}^{-1}$ cloud is reported in the region linking the Arc and the Sgr A complex (see below). The recombination line profiles are found to have large line widths ranging from $20 \mathrm{~km} \mathrm{~s}^{-1}$ to 100 $\mathrm{km} \mathrm{s}^{-1}$. It is not, however, clear if the large line widths are intrinsic and thus, characterize Galactic center features or are simply the result of the superposition of a number of velocity components. In this regard, it should be pointed out that the $-50 \mathrm{~km} \mathrm{~s}^{-1}$ and the $20 \mathrm{~km} \mathrm{~s}^{-1}$ molecular clouds and their possible HII counterparts show velocity dispersions which are few times higher than typical velocity dispersions found in the disk of the Galaxy.

b) $G 0.1+0.08$

G0.1+0.08, which is known as the arched filaments, the bridge, or the Arches, consists of a set of curved filaments which cross the nonthermal filaments of the radio continuum Arc at positive 


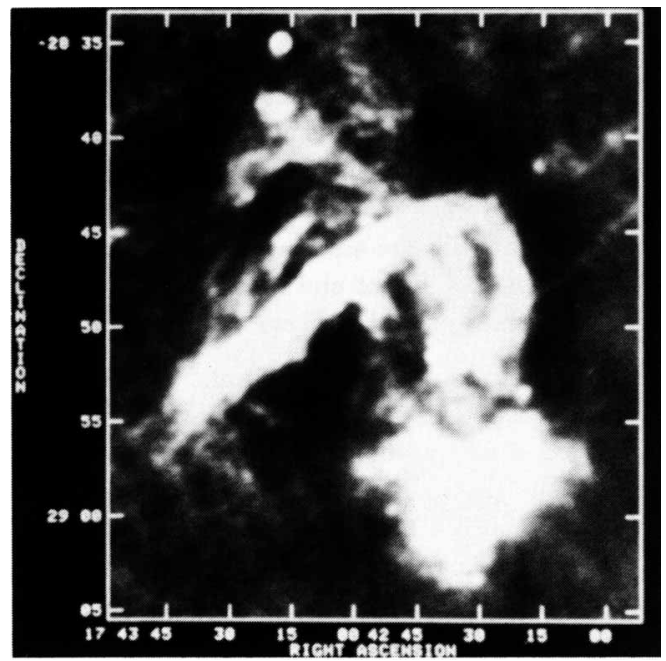

Figure 9: The $\lambda 20 \mathrm{~cm}$ radiograph of the Arc with a spatial resolution of $30^{\prime \prime} \times 30^{\prime \prime}$ shows a large-scale loop-like feature, G0.2-0.05. This feature appears to cross the arched filaments in northwest and G0.16-0.15 in southeast of the straight portion of the Arc.

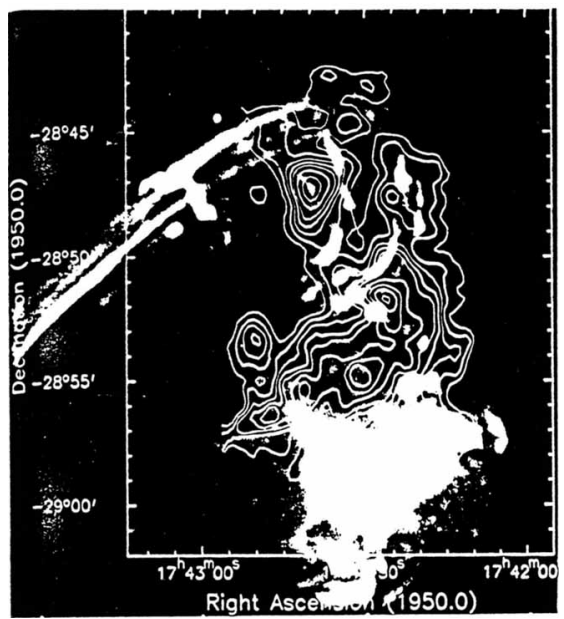

Figure 10: Contours of CS $\mathrm{J}=2 \rightarrow 1$ emission superimposed on the $\lambda 20 \mathrm{~cm}$ radiograph of the Arc and the Sgr A complex (Serabyn and Güsten 1987). 
latitudes. The velocity field of this source based on $\mathrm{H} 110 \alpha$ radio recombination line observations using the VLA indicates a continuous velocity structure which increases from $-50 \mathrm{~km} \mathrm{~s}^{-1}$ to $0 \mathrm{~km}$ $\mathrm{s}^{-1}$ northward along individual filaments (Yusef-Zadeh 1986; Yusef-Zadeh, Morris, and van Gorkom 1987). The direction in which the ionized gas flows along the filaments is not clear. The kinematics of thermal gas are unusual in that they are forbidden in the sense of Galactic rotation, i.e. negative velocity gas at positive longitude. Recent molecular CS, HI, and $10 \mu \mathrm{m}$ observations show clearly that the cool materials and dust particles are associated with the ionized gas (Serabyn and Güsten 1987; Lasenby et al. 1988; Yusef-Zadeh, Telesco and Decher 1988). The column density of molecular hydrogen is estimated to be between $10^{22}$ and $10^{23} \mathrm{~cm}^{-3}$.

Figure 10 is taken from Serabyn and Güsten (1987) and shows contours of CS emission in the range -55 to $5 \mathrm{~km} \mathrm{~s}^{-1}$ superimposed on the $\lambda 20 \mathrm{~cm}$ radiograph of the Arc and the Sgr A complex. The $-50 \mathrm{~km} \mathrm{~s}^{-1}$ molecular cloud is seen to lie predominantly at the edge of the thermal filaments and, unlike the distribution of the continuum emission, the cool materials are concentrated in the region between the Arc and the Sgr A complex. The spatial distribution of CS has led these authors to hypothesize that this cloud is as close to the Galactic center as it appears in projection. The recombination line survey finds an HII counterpart to the $-50 \mathrm{~km} \mathrm{~s}^{-1}$ molecular cloud and links the ionized gas in the arched filaments to that associated with the Sgr A complex (Anantharamaiah and Yusef-Zadeh 1988), and therefore, supports the suggestion made by Serabyn and Güsten. The total mass of the $-50 \mathrm{~km} \mathrm{~s}^{-1}$ molecular gas is estimated to be $\sim 6 \times 10^{5} \mathrm{M} \odot$ (Serabyn and Güsten 1987).

The ionization mechanism of the $-50 \mathrm{~km} \mathrm{~s}^{-1}$ molecular cloud is not understood. Since the the distribution of ionized gas is very different at the northern and southern boundaries of the $-50 \mathrm{~km}$ $\mathbf{s}^{-1}$ cloud, it is possible that different ionization mechanisms operate in different parts of this cloud. At the southern boundary, the proximity of the ionized gas to the Galactic center suggests that the ultraviolet radiation from Sgr A or the outflowing wind from the nucleus (YM87c) might be responsible for ionizing the southern edge of the cloud. At the northern boundary, the filamentary appearance of both thermal and nonthermal gas suggests that the magnetic structures might play a role in the ionization of the gas. Pictorial evidence in support of an interaction and the coupling between the thermal and nonthermal gas can be seen in Figure 11 (YM88). The panels on the left and right show the linearly polarized emission from the nonthermal filaments and the total continuum emission from the ionized filaments, respectively. The argument for the physical interaction, among other things, is based on the subtle appearance of a bend along the northernmost straight filaments. In this hypothesis, the magnetic field mediates the tranfer of energy to the neutral clouds by ohmic heating (Benford 1988; Morris and Yusef-Zadeh 1988). The strength of the magnetic field along the nonthermal filaments is estimated to be $\sim 2 \mathrm{mG}$ by assuming that the distortion is caused by the impact of the molecular gas and the nonthermal filaments. This value is an order of magnitude greater than that estimated by minimizing the sum of energies in the magnetic field and relativistic particles. The strength of the magnetic field along the thermal filaments is estimated to be $\geq 0.6$ $\mathrm{mG}$ assuming the ionized gas is in pressure equilibrium with the hot and diffuse $\mathrm{X}$-ray gas engulfing the Galactic center region (Heyvaerts, Norman and Pudritz 1988). Indeed, future IR polarization measurements of the hot and cold dust grains associated with the arched filaments and the -50 $\mathrm{km} \mathrm{s}^{-1}$ molecular cloud, respectively, are needed to examine the role of the magnetic field in the filamentary structure of G0.1+0.08.

An alternative interpretation for the ionization of the arched filaments is discussed by Genzel, Stacy and Townes (1988) who find a clear association between $\mathrm{C}^{+}$and HII gas along the arched filaments. These authors support the suggestion that the ionized gas is photoionized by external OB stars (see also Serabyn and Güsten 1987). 

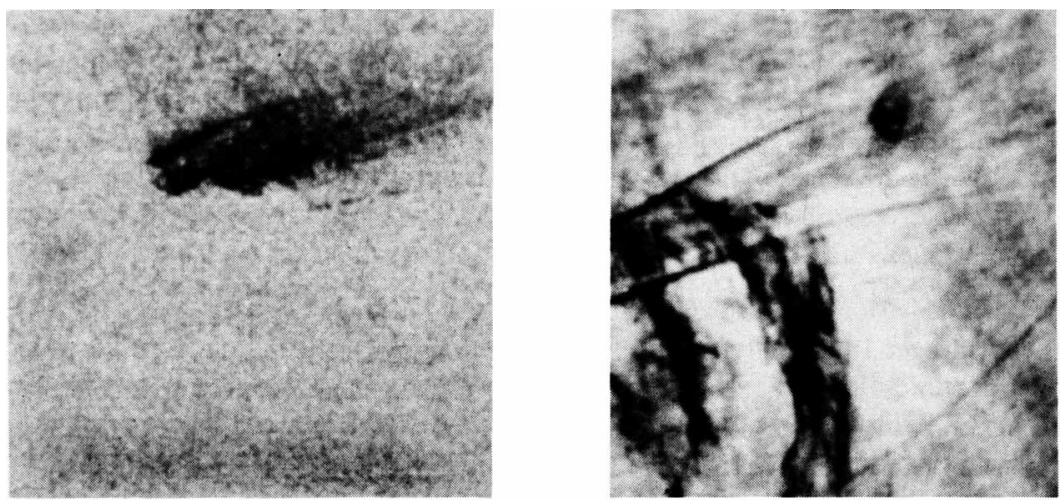

Figure 11: The polarized and total intensity distributions of the arched filaments at $\lambda 6 \mathrm{~cm}$ are shown in left and right side figures, respectively. The two images cover exactly the same area.

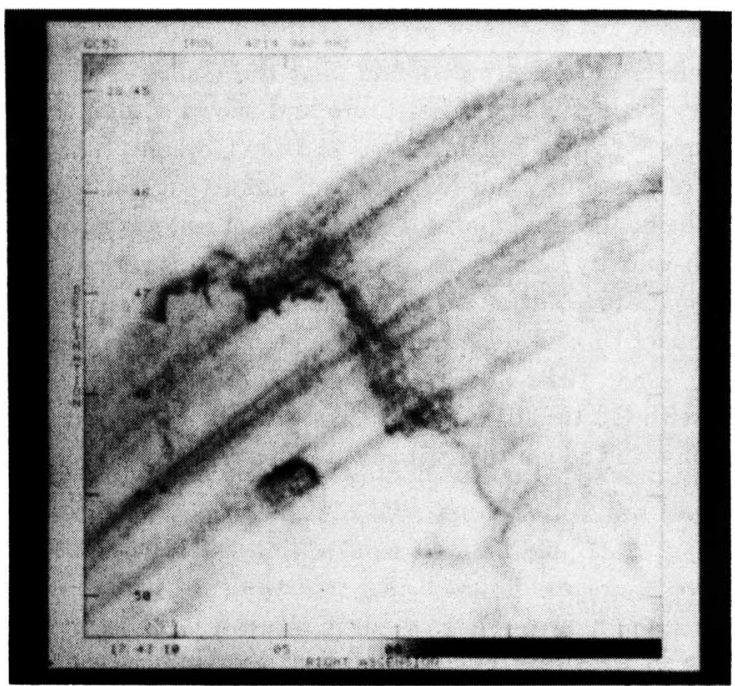

Figure 12: The total intensity radiograph of G0.18-0.04 at $\lambda 6 \mathrm{~cm}$ (YM87b). 


\section{b) $G 0.18-0.04$}

G0.18-0.04, which is nicknamed the "sickle", crosses the straight nonthermal filaments as they intersect the Galactic plane (see Fig. 12). The recombination line measurements indicate that the velocity of the ionized gas is predominantly between +20 and $+40 \mathrm{~km} \mathrm{~s}^{-1}$. The morphology of the nonthermal filament shows a change in their surface brightness and their linearity as they pass through the sickle feature. Based on these structural details, YM87b argue that an interaction between thermal and nonthermal gas may be responsible for the ionization of the sickle-shaped structure.

The physical association of the $40 \mathrm{~km} \mathrm{~s}^{-1}$ molecular cloud which is projected over the length of the Arc (Pauls and Mezger 1980; Bally et al. 1988 and YM87b) with the ionized features in G0.18-0.04 has recently come under some doubts: Recent $\mathrm{H} 92 \alpha$ recombination line measurements revealed the weakly emitting ionized gas associated with the sickle has high velocities of up to (not exclusively) $\sim 130 \mathrm{~km} \mathrm{~s}^{-1}$ (Yusef-Zadeh, Morris and van Gorkom, 1988). Also, a recent HI absorption study suggests that the $40 \mathrm{~km} \mathrm{~s}^{-1}$ molecular cloud lies behind the continuum Arc and therefore, may not be associated with G0.18-0.04, unless a special geometry is invoked (Lasenby et al. 1988). The HI absorption measurements indicate that the $20 \mathrm{~km} \mathrm{~s}^{-1}$ neutral cloud may be physically associated with the filaments since the presence of a gap in the distribution of HI gas coincides with the polarized region and with the peak emission at $160 \mathrm{MHz}$ near G0.16-0.15. Lasenby et al. argue that the $20 \mathrm{~km} \mathrm{~s}^{-1}$ gas may be responsible for most of the depolarization noted along the Arc except near G0.16-0.15 where the column density of gas associated with this cloud has a minimum.

One of the most curious thermal features found near the Galactic center is a compact source, G0.15-0.05, which lies very close to the sickle feature and shows a total linewidth of $\sim 90 \mathrm{~km} \mathrm{~s}^{-1}$ centered at $\mathrm{V}_{L S R} \sim 123 \mathrm{~km} \mathrm{~s}^{-1}$ (Yusef-Zadeh, Morris and van Gorkom, 1988). The unusual kinematical and spatial structure of this source has led these authors to suggest that low-energy relativistic particles associated with the nonthermal filaments heat the ambient gas clouds. Although no molecular counterpart to the high-velocity ionized gas has been seen in this region, the lack of emission may be due to relatively poor spatial resolution of the molecular line observations. Mid-infrared observations show a group of compact IR sources with a very high color temperature, $1000^{\circ} \mathrm{K}$, surrounding G0.15-0.05 (Okuda et al. 1988; Yusef-Zadeh, Telesco and Decher 1988). The true association of these unusual IR sources with G0.15-0.05 is not established, but it has been suggested that the high energy particles associated with the nonthermal gas are also responsible for heating the dust grains (Yusf-Zadeh, Telesco and Decher 1988).

\section{Discussion}

\section{a) Geometry of the Magnetic Field}

The overall distribution of the linear polarization near the Galactic center region suggests that the magnetic field is ordered on a scale of $\sim 200 \mathrm{pc}$ and has a poloidal geometry. Additional evidence in support of this hypothesis arises from the shape of Sgr A East shell, a prominent Galactic center nonthermal radio source. This source is thought to be produced as a result of an explosive event, possibly a supernova remnant (Goss et al. 1983; YM87c). It is elongated in the direction along the Galactic plane where the gas density is maximized. It is expected that the ram pressure in the Galactic plane would impede the ejected materials most in that direction unless the magnetic field plays a strong role in the dynamics of gas (Bernstein and Kulsurd 1965). A uniform magnetic field oriented perpendicular to the Galactic plane having a strength of $\sim 10^{-4} \mathrm{G}$ is sufficient to explain the elliptical shape of Sgr A East shell (YM87c). 
The morphological and kinematical evidence for the physical interaction between thermal and nonthermal features plus the appearance of an organized system of straight filaments which trace magnetic field lines with almost no distortion over a large expanse in a medium that is filled with a rich body of dense and clumpy molecular gas would imply that the strength of the magnetic field has to be of the order of $10^{-3} \mathrm{G}$ or greater. Similar field strengths are also derived to explain the large degree of IR polarization of the hot and cold dust grains detected in the inner few parsecs of the Galaxy (Aitken et al. 1986; Werner and Davidson 1988). The large pressure associated with the magnetic field along the filaments could dominate the the ambient gas pressure. Since there is no possibility of balancing the Lorentz force, the currents align themselves along the field lines in a force free configuration. A large strength of the magnetic field could have a direct effect on the rate of star formation in the Galactic center region (Morris 1988).

The dissipation of the large reservoir of magnetic energy, as a result of the relaxation of twisted magnetic field lines, could in principle balance the energy due to radiative losses of the linear filaments if the following assumptions are made: the field lines are anchored to the halo of the Galaxy, the twisting of the field lines are caused by the strong differential rotation, and the efficiency of converting magnetic energy to synchrotron radiation is $10^{-2}$ (YM87a).

The linear portion of the Arc and its northern and southern extensions are located in a region of the Galaxy where the differential rotation is strong. All the points along the Arc rotate at a constant angular velocity. However, based on the mass distribution in the central 200pc of the Galaxy, the Arc and its extensions, as a unified feature, cannot rotate with a constant angular velocity. Therefore, unless the lifetime of the Arc and its extensions are shorter than $10^{6}$ years, and unless the pressure associated with the field lines does not dominate the gas dynamics (see above), it is expected that the extensions of the Arc would lose their physical association with the linear segment of the Arc after a few rotation periods. Thus, it is possible that the flat spectrum of the linear filaments is an indication that the objects in question are young (Sofue et al. 1986; YM88).

\section{b) Relationship Between Neutral and Nonthermal Gas}

The possible association of the molecular clouds near the Galactic center and the magnetic structures has been suggested by numerous authors. This association is fueled by the appearance of the straight filaments in the Arc and G359.5+0.18 outlining the edge of prominent molecular clouds (Brown and Liszt 1984; Bally et al. 1988; Serabyn and Güsten 1987). The $40 \mathrm{~km} \mathrm{~s}^{-1}$ molecular cloud, as shown in Figure 13a, appears to lie at the edge of the nonthermal filaments. The $\mathrm{CO}$ and CS distributions and the nonthermal filaments in G359.54+0.18, as seen in Figure 13b, also show a similar behavior (Bally and Yusef-Zadeh 1989; Bally, Yusef-Zadeh and Hollis 1988). The magnetic field strength is estimated to be a few milli-Gauss by assuming that the magnetic pressure is in pressure equilibrium with that of the molecular gas. The implication of such a physical association is that the electric field generated as a result of the $\mathbf{v} \times \mathbf{B}$ term would be able to accelerate particles to high energies (see next section).

Due to the large concentration of massive molecular clouds lying toward the Galactic center, the association between the nonthermal filaments and the molecular clouds might be marred by chance superposition, and therefore, the true association is not well established; e.g. recent HI absorption and recombination line studies suggest the $20 \mathrm{~km} \mathrm{~s}^{-1}$ molecular cloud may be associated with the Arc and not the $40 \mathrm{~km} \mathrm{~s}^{-1}$ molecular cloud (see above discussion). Other circumstantial evidence which favors the hypothesis that the cold neutral material is associated with the magnetic structures are the definite physical association of the thermal filaments and the $-50 \mathrm{~km} \mathrm{~s}^{-1}$ molecular cloud, the strong evidence for the interaction of thermal and nonthermal filaments throughout the Arc, 

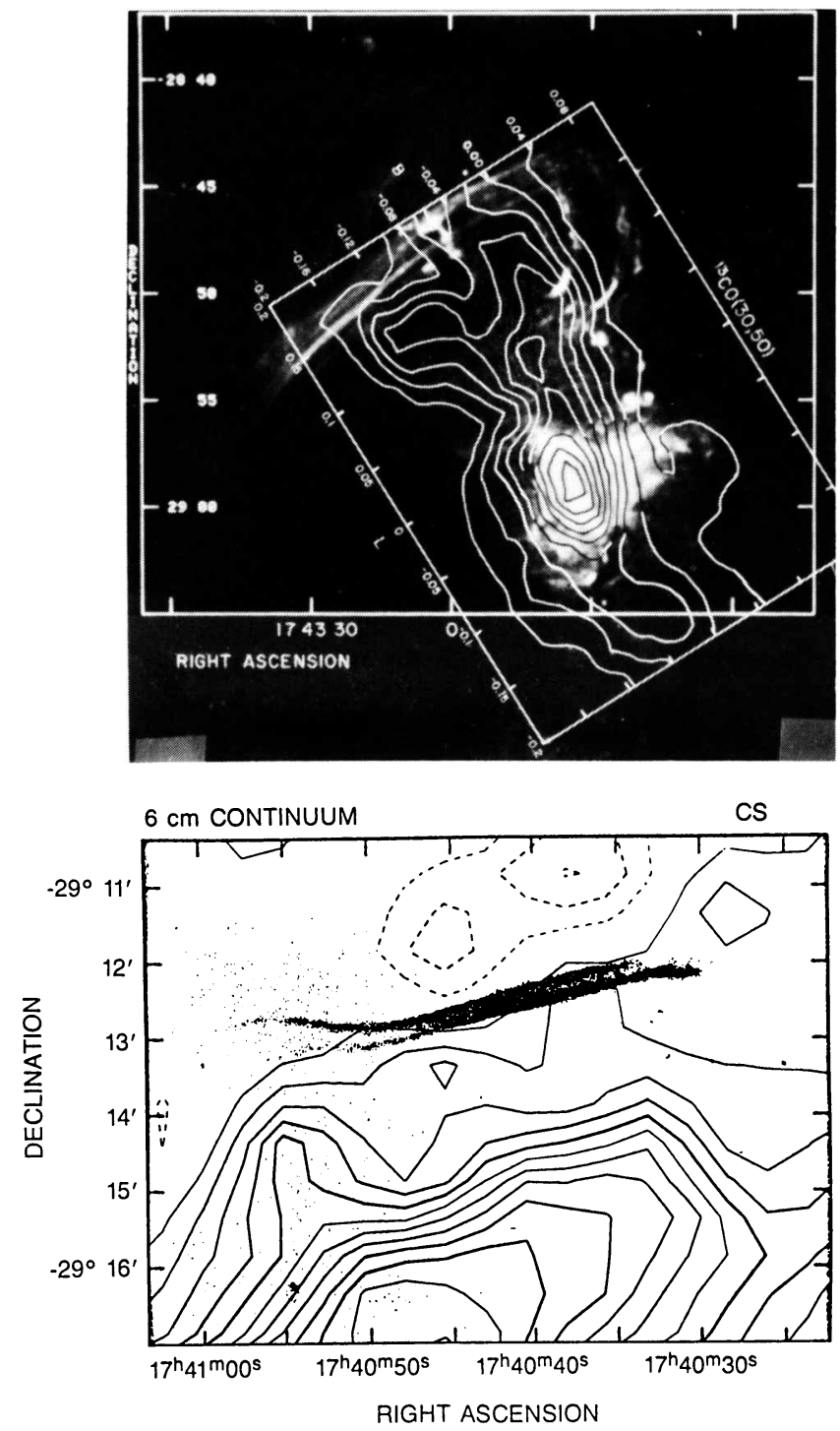

Figure 19a,b: Contours of ${ }^{13} \mathrm{CO}$ (Bally et al. 1988) and CS (Bally and Yusef-Zadeh 1989) emission integrated from $V_{L S R}=30$ to $50 \mathrm{~km} \mathrm{~s}^{-1}$ and -180 to $-80 \mathrm{~km} \mathrm{~s}^{-1}$ are superimposed on the $\lambda 20 \mathrm{~cm}$ and $\lambda 6 \mathrm{~cm}$ radiographs of the Arc and G359.54+0.18 and are shown in top and bottom figures, respectively. 
and the presence of a cool and dense absorbing filament at $\lambda 6 \mathrm{~cm}$ running adjacent to nonthermal filaments (see YM88 for more details). Future high-resolution observations of neutral material would be very important in elucidating the nature of the association between thermal and nonthermal gas.

\section{c) Origin of Filaments}

One of the key questions that has to be addressed is related to mechanisms which would accelerate particles to relativistic energies. A number of models have been proposed to explain some aspects of the observed phenomenon near the Galactic center. These models are described briefly below.

A: A detailed model by Benford (1988) shows that the large relative motion of molecular clouds with respect to preexisting magnetic field lines in the Galactic center would induce strong electric field $(\mathrm{v} \times \mathrm{B}) \mathrm{c}^{-1}$ of the order $10^{-8}$ statvolt $\mathrm{cm}^{-1}$ at the edge of the cloud which is sufficient to accelerate particles along the field lines, yielding synchrotron radiation. This model assumes the filamentary structures form a giant circuit in which current paths are illuminated by the passage of the particles. Anomalous resistivity is used to lower the conductivity, enhancing the diffusion of the magnetic field lines at the edge of the molecular clouds. The electric field is used locally to accelerate particles. However, it is not clear how these "batteries", i.e., local sources of heating, can align themselves to produce a large scale structure which extends over $200 \mathrm{pc}$.

A qualitative model by Bally et al. (1988) suggests that the fast moving molecular clouds produce a shock front which is responsible for the compression of the magnetized plasma. The acceleration of cosmic ray particles to high energies as a result of the compression would then illuminate the preexisting organized field structure. The nature of the acceleration mechanism is not clearly understood in this model. However, it is possible that the shock front provides the initial ionization needed at the edge of the molecular cloud before the induced electric field acts to accelerate particles. Alternatively, a recent model by Morris and Yusef-Zadeh (1988) suggests that Alfven critical ionization mechanism may be responsible for the partial ionization needed at the edge of the molecular cloud.

B: Heyvaerts, Norman and Pudritz (1988) hypothesized that the prominent radio structures including the Arc and its extensions are produced as a result of loop ejection from the Galactic center. They make an analogy between the "coronal loops" seen on the surface of the Sun and the Arc and have proposed the reconnection of the field as a driver to accelerate particles, unlike the model proposed by Benford (1988) who considers a local source as the cause of the observed phenomenon and microplasma instability, i.e. the anomalous resistivity, responsible for the decoupling of matter from the magnetic field lines. The MHD model of Heyvaerts et al. relies on the assumption that a centrally differentially rotating engine at the Galactic center expels magnetic loops and the reconnection accelerates particles to high energies. The morphology of G359.54+0.18 supports the reconnection hypothesis as a means to accelerate particles (Bally and Yusef-Zadeh 1989). G359.54+0.18 consists of two sets of straight filaments which show an increase in their surface brightness as the distance between the filaments decrease.

C: Shibata and Uchida (1987) have considered the possibility that the "omega-shaped" structure is formed as a result of the contraction of a rotating nuclear disk in a medium threaded by the largescale poloidal magnetic field. As the materials rotate and contract, the field lines get twisted and the gas in the disk propagates along the magnetic field in the direction away from the Galactic plane. The numerical simulation reproduces the large-scale "omega-shaped" structure and the opposite sign of the line-of-sight component of the the magnetic field along the extensions of the Arc. However, it is not clear how this three-dimensional model could account for the morphology of the nearly straight filaments near the Galactic plane and the one-dimensional appearance of the "threads". 
D: Sofue and Fujimoto (1987) proposed a model in which the supersonic thermal materials are ejected from the nucleus and are distorted as they encounter the poloidal magnetic field. In this model, the bright ionized structures along the arched filaments are produced as the result of a shock front. Recent $2 \mu \mathrm{m}$ spectroscopy along the arched filaments failed to detect molecular hydrogen line emission and, therefore, rules out the possiblity that the gas is shock heated (Geballe, Serabyn and Yusef-Zadeh 1988).

E: It has been suggested that the threads may be related to cosmic strings, which are hypothesized remnants of the early universe, and which are presumed to help nucleate galaxy formation (Vilenkin 1985). According to some theories, strings should be superconductors which as they oscillate at nearly the speed of light through a magnetic medium create an intense magnetohydrodynamical interaction (Chudnovsky et al. 1986). If the threads are manifestation of the interaction between strings and the magnetized plasma near the Galactic center, the measurement of proper motion would be able to test this hypothesis.

We wish to thank Dr. R.J. Oliversen for his critical reading of this manuscript.

\section{References}

Aitken, D.K., Roche, P.F., Bailey, J.A., Briggs, G.P., Hough, J.H. and Thomas, J.A. 1986, MNRAS, 218, 363.

Altenhoff, W.J., Downes, D., Pauls, T. and Schraml, J. 1978, Astr.Astrophys.Suppl., 35, 23.

Anantharamaiah, K.R. and Yusef-Zadeh, F. this volume.

Bally, J., Stark, A., Wilson, R. and Henkel, C. 1988, Ap.J., 324, 223.

Bally, J. and Yusef-Zadeh, F. 1989, Ap.J. (January 15 issue).

Bally, J., Yusef-Zadeh, F. and Hollis, J.M. this volume.

Benford, G. 1988, Ap.J. (in press).

Bernstein, I.B., and Kulsrud, R.M. 1965, Ap.J., 142, 479.

Brown, R.L. and Liszt, J.H. 1984, Ann.Rev.Astron.Astrophys., 22, 223.

Burn, B.F. 1966, Ap.J., 324, 223.

Chudnovsky, E.M., Field, G.B., Spergel, D.N. and Vilenkin, A. 1986, Phys.Rev.D., 34, 944.

Dent, W.A., Werner, M.W., Gatley, I., Hildebrand, R.H., Keene, J. and Whitcomb, S.E. 1982,

The Galactic Center, eds. G.R. Reigler, R.D. Blandford, (New York:AIP), p33.

Drake, F. 1959, Astr.J., 64, 329.

Ekers, R., van Gorkom, J.H., Schwarz, U.J. and Goss, W.M. 1983, Astron.Astrophys., 122, 143.

Geballe, T.R. 1987, The Galactic Center, ed. D.C. Backer (New York:AIP), p39.

Geballe, T.R. this volume.

Geballe, T.R., Serabyn, E., and Yusef-Zadeh, F. 1989, in preparation.

Genzel, R., Stacy, G.J. and Townes, C.H. this volume.

Goss, W.M., Schwarz, U.J., Ekers, R.D. and van Gorkom, J.H. 1983, IAU Symposium 101, Supernova Remnants ans their X-ray Emission, eds. J. Danziger and P. Gorenstein, (Dordrecht:Reidel), p65.

Güsten, R. and Downes, D. 1980, Astron.Astrophys., 87, 6.

Heyvaerts, J., Norman, C. and Pudritz, R. 1988, Ap.J., (in press).

Inoue, M., Fomalont, E., Tsuboi, M., Yusef-Zadeh, F., Morris, M., Tabara,H. and Kato, $\mathrm{T}$. this volume.

Inoue, M., Takahashi, T., Tabara, H, Kato, T. and Tsuboi, M. 1984, Pub.Astr.Soc.Japan., 36, 633 . 
Kapitzky, J.E. and Dent, W.A. 1974, Ap.J., 188, 27.

Kassim, N.E., Erickson, W.C. and LaRosa, T. 1987, The Galactic Center, ed. D.C. Backer (New York:AIP), p196.

Lasenby, J., Lasenby, A.N. and Yusef-Zadeh, F. this volume.

Liszt, H.S. 1985, Ap.J.(Letters), 239, L65.

Little, A.G. 1974, IAU Symposium 60, Galactic Radio Astronomy, ed. F.J. Kerr and S.C. Simonson III, (Dordrecht:Reidel), p491.

Mezger, P.G., Zylka, R., Salter, C.J., Wink, J.E., Chini, R. and Kreysa, E., this volume. Mills, B.Y., and Drinkwater, M.J. 1984, J.Ap.Astr., 5, 43.

Morris, M. this volume.

Morris, M. and Yusef-Zadeh, F. 1985, Astron.J., 90, 2511.

Morris, M. and Yusef-Zadeh, F. 1988, submitted to Ap.J.

Morris, M. and Yusef-Zadeh, F. 1989, in preparation

Odenwald, S.F. this volume.

Okuda, H., Shibai, H., Matsuhara, H., Kobayashi, Y., Kaifu, N. et al. this volume.

Pauls, T., Downes, D., Mezger, P.G., Churchwell, E., 1976, Astron. Astrophys. 46, 407.

Pauls, T., Mezger, P.G., 1980, Astron. Astrophys., 85, 26.

Reich, W. this volume.

Reich, W., Sofue, Y. and Fürst, E. 1988, preprint.

Seiber, W. and Schlickeiser, R. 1982, Astron.Astrophys., 113, 314.

Seiradakis, J.H., Lasenby, A.N., Yusef-Zadeh, F., Wielebinski, R. and Klein, U. 1985, Nature, 317, 697.

Serabyn, E. and Güsten, R., 1987, Astron.Astrophys., 184, 183.

Shibata, K. and Uchida, Y. 1987, Publ.Astron.Soc.Japan, 39, 559.

Sofue, Y. and Fujimoto, M. 1987, Ap.J.(Letters), 319, L73.

Sofue, Y. and Handa, T. 1984, Nature, 310, 568.

Sofue, Y., Inoue, M., Handa, T., Tsuboi, M., Hirabayashi, H., Morimoto, M. Akabane, K., 1986, Publ. Astron. Soc. Japan, 38, 475.

Sofue, Y., Reich, W., Inoue, M. and Seiradakis, J.H. 1987, Pub.Astr.Soc.Japan., 39, 95.

Steinberg, J.L. and Lequeux, J. 1963, Radio Astronomy, (McGraw Hill, New York), p193.

Tsuboi, M., Inoue, M., Handa, T., Tabara, H. and Kato, T., Sofue, Y. and Kaifu, N. 1986, Astron.J., 92, 818.

Vilenkin, A. 1985, Phys.Rep., 121, 263.

Werner, M.W. and Davidson, J. this volume.

Whiteoak, J.B. and Gardner, F.F. 1973, Astrophys.Lett., 13, 205.

Wright, A.E. and Barlow, M.J. 1975, MNRAS, 170, 41.

Yusef-Zadeh, F., 1986, Ph.D Thesis, Columbia University.

Yusef-Zadeh, F. and Bally, J. 1989, in preparation.

Yusef-Zadeh, F., Morris, M. 1987a, Ap.J., 322, 721.

- 1987b, Astron.J., 94, 1128.

1987c, Ap.J., 320, 545.

1988, Ap.J., 329, 729 .

Yusef-Zadeh, F., Morris, M., Chance, D. 1984, Nature, 310, 557.

Yusef-Zadeh, F., Morris, M., Lasenby, A., Seiradakis, J.H., Wielebinski,

R. and Klein, U. 1989, in preparation.

Yusef-Zadeh, F., Morris, M., Slee, O.B. and Nelson, G.J. 1986, Ap.J., 310, 689.

Yusef-Zadeh, F., Morris, M., and van Gorkom, J.H. 1987, in The Galactic Center, ed.

D.C. Backer (New York:AIP), p190.

Yusef-Zadeh, F., Morris, M., and van Gorkom, J.H. this volume.

Yusef-Zadeh, F., Telesco, C. and Decher, R. this volume. 